\title{
Aufgaben der Ergotherapie im Rahmen der Behandlung psychisch Kranker
}

\author{
Hermann Mecklenburg \\ Psychiatrische Klinik I, Kreiskrankenhaus Gummersbach
}

psychoneuro 2003, 29 (4): 184-186

$\mathrm{E}$ rgotherapie ist in der stationären psychiatrischen Behandlung, die die regionale Grundversorgung sicherstellt, außer der ärztlichen Tätigkeit das am weitesten verbreitete therapeutische Verfahren. Dafür gibt es gute Gründe:

- Ergotherapie ist ein handlungsorientieres Verfahren

- sie besitzt ein breites Indikationsspektrum

- $\quad$ sie bietet ein differenziertes methodisches Repertoire.

Ergotherapie würde zu Recht diese Bedeutung auch in der ambulanten psychiatrischen Behandlung einnehmen in der Weise, wie das z.B. die Physiotherapie für die Orthopädie besitzt. Wenn auch noch regional sehr unterschiedlich, so wächst doch ambulante Ergotherapie als Heilmittel der ärztlich-psychiatrischen Behandlung allmählich zu. Es gibt inzwischen Praxen, die sich einen psychiatrischen Schwerpunkt gebildet habe. Im institutionellen Rahmen kann ambulante Ergotherapie in Trägerschaft psychiatrischer Kliniken oder im gemeindepsychiatrischen Kontext stattfinden. Jede dieser Versorgungsformen steht dem niedergelassenen Nervenarzt zur Mitbehandlung für seine Patienten offen.

\section{- Handlungsorientierung}

Die Psychiatrie gilt gemeinhin als sprechendes Fach. Anders als in den übrigen Fächern nimmt die medizinische Behandlung und Diagnostik im engeren Sinne nur wenig Raum ein,

Ergotherapie erhält eine zunehmend bedeutsame Rolle in der psychiatrischen Therapie: Ergotherapie ist handlungsorientiert, besitzt ein weites Indikationsspektrum und bietet eine breite Palette unterschiedlicher Methoden. Psychiatrische Ergotherapie ist gegenwärtig zwar noch stärker im Krankenhaus verankert, aber es ist zu erwarten, dass sie auch in der ambulanten Behandlung eine herausragende Funktion einnehmen wird als ergänzende Therapie zur nervenärztlichen Behandlung (vergleichbar der Physiotherapie in der Orthopädie) - die übrigen Beiträge dieser Serie zeigen das auf. Diese Arbeit exemplifiziert den handlungsorientierten Charakter der Ergotherapie im Allgemeinen (in Ergänzung zur ärztlich psychiatrischen und zur Psychotherapie) und der Arbeitstherapie als Mittel zur Hinführung zu Beschäftigung und Arbeit im Speziellen. Es werden die Felder aufgezeigt, in denen Ergotherapie in der psychiatrischen Behandlung ihre spezifische Kompetenz entfaltet.

wenn der Mensch im Krankenhaus ist. Der Patient liegt nicht im Bett. Er ist nicht krank in der Form, die ihm aus seiner Alltagserfahrung geläufig ist. Die Mitarbeiter verhalten sich in der Regel nicht wie „normale“ Ärzte und Schwestern. Sie tragen oft keine Kittel, sind nicht unbegrenzt fürsorglich, manchmal sogar streng und fordernd und verlangen z.B. vom Patienten, er solle selbst sein Bett machen. Und das, was er aufgrund allgemeinen Wissens von der sprechenden Medizin erwartet, nämlich Einzelgespräche täglich und ausreichend lange, wird auch nicht in dem erwünschten Maße geboten. Tatsächlich - so findet der Patient bald heraus - ist die Psychiatrie in der Akutbehandlung nicht überwiegend ein sprechendes Fach. Aber auch keines, welches ihn von eigener HandlungsVerantwortung weitgehend freistellt wie die Krankenhausbehandlung in der somatischen Medizin.

Was aber dann? Für den Patienten addieren sich in dieser Situation psychiatrische Symptome und Rollenverunsicherung - vor allem zu Beginn, aber auch noch über lange Zeiten während des stationären Aufenthaltes.

In der nervenärztlichen Praxis verhält es sich ähnlich: Arzt und $\mathrm{Pa}$ tient interagieren nach dem Modell der somatischen Medizin. Raum fürs Sprechen ist oft nur wenig da. Entweder weil die Indikation nicht gegeben ist oder weil die Struktur unseres medizinisch-ambulanten Versorgungssystems das nur eingeschränkt erlaubt. Und beide - Arzt und Patient - wissen, dass dieses medizinische Modell für ihre Begegnung nur eingeschränkt passt. Trotz gutem Kontakt zwischen Arzt und Patient fehlt oft etwas.

Das Modell der psychotherapeutischen Medizin ist ebenfalls nur selten anwendbar. In sehr kurzer Form ist dieses Modell wie folgt zu skizzieren:

Symptome sind Ausdruck innerer Konflikte, die anders als mit die- 
sen Symptomen nicht mehr angstfrei zu lösen waren. Im aufdeckenden und klärenden Sprechen wird die Verbindung zwischen Symptom und Konflikt verstehbar gemacht, und im durcharbeitenden Sprechen werden Lösungen erarbeitet, die der Patient dann im wirklichen Leben ausprobiert und umsetzt.

Dieses Modell der sprechenden Medizin gilt nur eingeschränkt

- für Menschen, die sich in psychiatrischer Akutbehandlung befinden

- für Menschen, für die Sprechen außer in Bezug auf alltägliche konkrete Dinge eher ungewohnt ist

- für Menschen, die sich beim Denken eher nicht im Abstrakten oder auf wechselnden Ebenen bewegen

- für Menschen mit schweren Krankheitsverläufen, die hauptsächlich damit zu tun haben, einigermaßen angstfrei durchs Leben zu kommen.

Schließlich stellt sich heraus: Die Psychiatrie ist oft kein sprechendes Fach. Die Psychiatrie ist in vielen Aspekten kein medizinisches Fach im engeren Sinne. Die Psychiatrie ist ein handelndes Fach.

Und hier kommt nun die Ergotherapie ins Spiel. Die Ergotherapie ist die Behandlungsform, die methodisch und von den Indikationen her das weiteste Spektrum als „Tätigsein im Behandeln“, als „Behandeln durch Tätigsein“ entwickelt hat (8, 9). Tätigsein ist eine integrierende Funktion menschlicher Existenz (1). Wir sehen die spezifischen Folgen von Arbeitslosigkeit nicht nur, weil dann Geld und soziale Anerkennung fehlen (6). Tätigsein-Wollen ist ein menschliches Urbedürfnis wie Hunger, Sexualität und Lernbegier (4). Pflegedürftige, die unbeweglich im Bett liegen, verlieren die körperliche Identität, spüren ihre Grenzen nicht mehr, alles zerfließt ins Wolkige und Unkonkrete. Fehlendes Tätigsein löst Ähnliches beim Menschen und seiner psychischen Identität aus: Zeitgrenzen und Leistungsgrenzen lösen sich auf. Der Raum wird in der Wahrnehmung diffus, wenn wir ihn nicht im Tätigsein zielgerichtet durchmessen.
Psychotisches Erleben wird gefördert (2). Langeweile kann zwar positiv gewendet werden zu „langer Weile“ als Zustand von Ruhe und Gelassenheit. Es erfordert aber Kraft und Weisheit, diesen Schritt zu tun. Sonst erzeugt Langeweile die Wahrnehmung von Leere - ein klassischer Auslöser für Angst und Depression und wiederum Psychose.

Der entscheidende Wirkfaktor der Ergotherapie ist Behandlung durch Handlung. Dieses Konzept ist in seinen Grundzügen sehr alt. Hinweise auf Behandlung durch Beschäftigung finden sich im Altertum bereits bei Asklepios und Galen. In der modernen Psychiatrie geht die historische Linie von dem „Non Restraint“-Ansatz im England des 18. Jahrhunderts über Pinél, Reil und Griesinger im 19. Jahrhundert bis zu Hermann Simon in den 20-er Jahren des vorigen Jahrhunderts. Die Konzeptualisierung der „Occupational Therapy“ begann Anfang des 20. Jahrhunderts in den USA (die erste Schule für Ergotherapie wurde 1908 in Chikago gegründet); die Einführung und dann allmähliche Ausbreitung fand in Deutschland nach dem 2. Weltkrieg statt.

\section{Indikationsspektrum und Ziele der Ergotherapie}

Beispielsweise nach den Krankheitsstadien (9) kann wie folgt vorgegangen werden. Menschen in sehr akuten Krisen nutzen Ergotherapie z.B.:

- zur Bindung von Angst durch Konzentration aufs Handeln

- zum Ordnen des Denkens durch zielgerichtetes Handeln

- zur Wahrnehmung von Grenzen durch berührendes Handeln

- zur Gewinnung von Ruhe durch stetiges Handeln.

Die dazugehörige Methode ist am ehesten die „kompetenzorientierte“ (zu den Methoden siehe den Beitrag von Frau Döring im nächsten Heft).

Menschen in abklingenden Krisen nutzen die Ergotherapie zusätzlich für den Wiedergewinn und den Zugewinn von Beziehungsmöglichkeit durch gemeinsames Handeln. Die dafür hinzutretende Methode ist die „interaktionelle“. Menschen mit abgeklungenen Krisen nach Psychoseerkrankungen und Patienten mit Neurose- und Suchtkrankheiten in abklingender Krise nutzen die Ergotherapie zusätzlich zur Wahrnehmung und Auseinandersetzung mit inneren Gefühlen, Wünschen und Strebungen. Die dafür hinzutretende Methode ist die „ausdruckszentrierte“.

Die Indikation für die jeweils nächstgenannte Methode und parallel dazu das Abklingen der akuten Symptomatik gehen beim Patienten einher mit einem Zugewinn an IchStärke, der den Einsatz der jeweils nächsten - d.h. psychisch anspruchsvolleren - Methode gestattet. Aber auch die vorher genannten Methoden behalten ihr Recht und bleiben indiziert mit den weitergehenden Zielen, z.B. Stabilisierung und Verbesserung der beruflichen und lebenspraktischen Fähigkeiten mit Hilfe der kompetenzorientierten Methode, oder Erweiterung von Kritik- und Konfliktfähigkeit mit Hilfe der interaktionellen Methode.

\section{Arbeitsbezogene \\ Ergotherapie}

Die Arbeitsbezogene Ergotherapie hat ihre Ursprünge in der $\mathrm{Ar}$ beitstherapie der psychiatrischen Fachkrankenhäuser. Unglücklicherweise wurde manchmal das Kind mit dem Bade ausgeschüttet, als in den 70-er und 80-er Jahren des letzten Jahrhunderts die ausbeuterischen Seiten des Systems Arbeitstherapie mit ihren „Hoftrupps“ und „Schälküchen“ erkannt wurden und ganze Arbeitstherapieabteilungen abgeschafft wurden. An einigen Orten ist die klinische Arbeitstherapie wieder zu Blüte gelangt mit modernen Inhalten und Strukturen und nach außen gerichteten Ansätzen (7). Die Diskussion ist allerdings im Gange, ob das so sein soll und ob die Kliniken sich dieses Feldes wieder stärker annehmen sollen oder ob nicht auch dieser Behandlungsansatz in gemeindepsychiatrische und rehabilitative ambulante Kontexte gehört (3, $5)$. Wie immer die Praxis sich entwickeln wird, die psychiatrischen Kliniken haben eher eine zunehmende Verpflichtung, ihren Patienten Wege zu weisen zu Arbeit und 
Beschäftigung. Möglicherweise ist dieser Beratungs- und Begleitungsauftrag in einem vernetzten gemeindepsychiatrischen System die verbleibende Aufgabe der sich auf die Akutbehandlung reduzierenden psychiatrischen Krankenhäuser.

Seit einiger Zeit rückt das Thema „Arbeit und Beschäftigung“ in der Gemeinde- und Sozialpsychiatrie in den Vordergrund, und die arbeitsbezogene Ergotherapie entwickelt sich zu einer zentralen Behandlungsform für die Hinführung zur medizinischen und beruflichen Rehabilitation oder zur Vorbereitung auf eine Beschäftigung. Insbesondere Assessment und Belastungserprobung als diagnostische Instrumente, aber auch arbeitsbezogene Ergotherapie im Rahmen der ambulanten Behandlung sowie der Rehabilitation sind bewährte und wachsende Aufgabenfelder (10). Allerdings findet dieses nun tatsächlich weniger in den Kliniken statt, sondern mehr und zunehmend im ambulanten Kontext: In Praxen und sozialpsychiatrischen Zentren, die ihre Klienten wiederum begleiten hin zur beruflichen Rehabilitation und Integration oder zu niederschwelligen Beschäftigungsinitiativen.

Es schließt sich hier der Kreis zu den vorherigen Ausführungen zur Bedeutung vom Tätigsein. Die Frage nach Arbeit und Beschäftigung muss in Zukunft mehr als bisher in therapeutischen und betreuenden Kontexten gestellt werden. Diese Frage muss für die Lebensplanung eines jeden Klienten beantwortet werden. Und es muss Beschäftigungsangebote geben nach den Bedürfnissen und Fähigkeiten der Klienten. Wenn ich schreibe „muss“, dann meine ich nicht irgendeinen anonymen $\mathrm{Ge}$ setzgeber oder Kostenträger, sondern uns selbst. In jeder Tagesstätte z.B. könnten ohne Mühe und weiteres Personal einige Zuverdienstplätze eingerichtet werden für Menschen, die ein oder zwei Stunden am Tage arbeiten, nicht jedoch den ganzen Tag betreut verbringen wollen und dieses auch nicht brauchen. Die Ergotherapeuten sind am ehesten sensibilisiert und haben vor anderen einen Blick für dieses Lebensfeld unserer Patienten und Klienten.
Gemeinsam müssen wir erkennen dass auch sozial- und gemeindepsychiatrische Betreuungsangebote Chronifizierung fördern - und dass Arbeit und Beschäftigung dem vorbeugen können.

\section{Einsatzfelder der Ergotherapie}

- Psychiatrische und psychotherapeutische Behandlung stationär, teilstationär, nachstationär und ambulant

- Medizinische Rehabilitation

- Berufliche Rehabilitation

- Sozialpsychiatrische Betreuung im Heim, in der Tagesstätte und in der Kontaktstelle

- Soziotherapie

- Arbeit

- Integrationsfachdienst

- Integrationsbetriebe.

Ergänzende Arbeitsfelder können über Weiterbildung erschlossen werden, die sich organisch an die ergotherapeutische Grundqualifikation anknüpfen lassen. Hier sind vor allem die weiteren „kreativen Therapien“ zu nennen: Musiktherapie, Tanztherapie und Kunsttherapie. Für die mir bekannten Behandlungsfelder und die mir anvertrauten Patienten wünsche ich mir eher integrierte Qualifikationen in der Gruppe der Ergotherapeuten als vielfältige Sondertherapeuten mit dem Risiko, dass über Konkurrenzgebahren Reibungsverluste entstehen.

\section{Resümée}

Es wird aus diesen Ausführungen deutlich: ErgotherpeutInnen können nach Herkommen und beruflichen Möglichkeiten in der Psychiatrie neben den Ärzten und den Pflegekräften die Generalisten in der Behandlung und Betreuung psychisch kranker Menschen sein.

\section{Summary}

Occupational therapy ist becoming a more and more important part of psychiatric therapy. It is acivity oriented, bears a wide range of indications und offers a great variety of methods. Although at present it is being used mainly in inpatient therapy, we expect occupational therapy to gain a more significant position in outpati- ent therapy as well, complementing psychiatric therapy - in a way physiotherapy complements orthopedic surgery. This will be demonstrated in the other papers of this issue. This paper illustrates the activity oriented character of occupational therapy in general and of industrial therapy in particular. It will point out those fields of medical care in which cupational therapy unfolds its specific competence.

\section{Key Words}

Occupational therapy - psychiatric therapy - industrial therapy

\section{Literatur}

1. Arendt H. Vita Activa oder vom tätigen Leben. München, Piper, 1981

2. Bennet D. Die Bedeutung der Arbeit für die psychiatrische Rehabilitation. In: von Cranach M, Finzen A (Hrsg). Sozialpsychiatrische Texte. Psychische Krankheit als sozialer Prozess. Psychiatrische Epidemiologie. Berlin/Heidelberg, Springer, 1972, 68-78

3. Fasshauer $\mathrm{K}$, Haerlin $\mathrm{C}$, Mecklenburg $\mathrm{H}$. Berufliche Rehabilitation psychisch Kranker und Behinderter in funktionaler Betrachtungsweise. Rehabilitation 1999; 38: 44-46 4. Ginsburg H, Opper S. Piagets Theorie der geistigen Entwicklung. Stuttgart, KlettCotta, 1975

5. Haerlin C, Mecklenburg H. Auf dem Weg zu personenzentrierten Hilfen zur Teilhabe am Arbeitsleben - Erfahrungen aus zwei rheinischen Regionen. In: Aktion Psychisch Kranke, Schmidt-Zadel R, Pörksen N (Hrsg): Teilhabe am Arbeitsleben. Arbeit und Beschäftigung für Menschen mit psychischen Beeinträchtigungen. Bonn, Psychiatrie Verlag, 2002, 259-277

6. Jahoda M. Wieviel Arbeit braucht der Mensch? Weinheim, Beltz, 1983

7. Mecklenburg H. Zwölf Thesen für eine langfristig erfolgreiche erfolgreiche berufliche Integration psychisch Kranker und Behinderter. Psychiatrische Praxis 1999; 26: 227-232

8. Scheepers C, Steding-Albrecht U, Fehn P. Ergotherapie - vom Behandeln zum Handeln. Stuttgart/New York, Thieme, 1999

9. Scheiber I. Ergotherapie in der Psychiatrie. München, Bardtenschlager, 1995

10. Weber P, Steier F. Arbeit schaffen. Initiativen, Hilfen, Perspektiven für psychisch Kranke. Bonn, Psychiatrie Verlag, 1998

\section{Korrespondenzadresse:}

Dr. H. Mecklenburg

Chefarzt Psychiatrische Klinik I

Kreiskrankenhaus Gummersbach

Wilhelm-Breckow-Allee 20

51643 Gummersbach 